\title{
Sugar does not affect the bending and tilt moduli of simple lipid bilayers
}

\author{
John F. Nagle*, Michael S. Jablin, and Stephanie Tristram-Nagle \\ Department of Physics, Carnegie Mellon University, Pittsburgh, PA 15213 USA
}

$12 / 16 / 2015$

\begin{abstract}
The diffuse X-ray scattering method has been applied to samples composed of SOPC, DOPC, DMPC, and POPC with added sugar, either sucrose, glucose, fructose, maltose, or trehalose. Several sugar concentrations in the range $200-500 \mathrm{mM}$ were investigated for each of the lipid/sugar samples. We observed no systematic change in the bending modulus $\mathrm{K}_{\mathrm{C}}$ or in the tilt modulus $\mathrm{K}_{\theta}$ with increasing sugar concentration. The average values of both these moduli were the same as those of the respective pure lipid controls within statistical uncertainty of $2 \%$. These results are inconsistent with previous reports of sugar concentration dependent values of $\mathrm{K}_{\mathrm{C}}$.
\end{abstract}

* Corresponding author nagle@ cmu.edu and 412-268-2764

Keywords: Mechanical properties; bending modulus; tilt modulus; lipid bilayers. 


\section{Introduction}

The bending modulus $\mathrm{K}_{\mathrm{C}}$ is a fundamental mechanical property of membranes that has overarching biophysical relevance. It has been a concern (Nagle et al., 2015, Nagle, 2013) that, even for simple lipid bilayers, there are significant differences in the reported values for $\mathrm{K}_{\mathrm{C}}$. An hypothesis for the disparate values is that the true bending modulus may not only be a property of the intrinsic lipid bilayer but may also vary upon adding sugar to the aqueous environment. If true, this would affect the values of $\mathrm{K}_{\mathrm{C}}$ determined by the classical methods of shape analysis (Meleard et al., 1998, Henriksen and Ipsen, 2002, Meleard et al., 1997, Pecreaux et al., 2004, Gracia et al., 2010, Bouvrais, 2012, Vitkova and Petrov, 2013) and mechanical manipulation (Rawicz et al., 2000, Henriksen and Ipsen, 2004, Vitkova et al., 2006, Shchelokovskyy et al., 2011, Evans and Rawicz, 1990). Both methods have typically used sugar to improve optical contrast. The disparity in $\mathrm{K}_{\mathrm{C}}$ values could then have arisen because different studies have used different sugar concentrations. In this report we test this hypothesis using the method of low angle diffuse $\mathrm{x}$-ray scattering from oriented stacks of membranes to measure $\mathrm{K}_{\mathrm{C}}$ (Lyatskaya et al., 2001, Liu and Nagle, 2004, Salditt et al., 2003, Li et al., 2006, Pan et al., 2008, Pan et al., 2009, Jablin et al., 2014). The most recent extension of this method also determines the tilt modulus $\mathrm{K}_{\theta}$ (Jablin et al., 2014, Jablin, 2015), so we report the effect of sugar on this modulus that only our method has been able to determine experimentally. We conclude by briefly discussing why the shorter length scale probed by x-rays compared to the classical methods is unlikely to alter our conclusion that sugar has no effect on true bending moduli.

\section{Experimental methods}

Lipids studied were SOPC, DOPC, DMPC, and POPC obtained from Avanti Polar Lipids. Sugars studied were sucrose, glucose, fructose, maltose, and trehalose obtained from Sigma/Aldrich. Samples were made by first mixing lipid and sugar (first solubilized in heated trifluoroethanol or methanol) in excess 1:1 vol:vol chloroform/(trifluoroethanol or methanol) organic solvent. Mole ratios of sugar to lipid $\mathrm{n}_{\mathrm{S}}$ are given in Table 1. The mixtures were then deposited on Si wafers using the rock and roll technique, creating stacks of about 2000 aligned bilayers (Tristram-Nagle, 2007). 
Dry samples were then hydrated in a humidity chamber in situ on the x-ray beamline. Hydration was conveniently even more rapid and, importantly, proceeded further with sugar than for pure lipid. Table 1 shows the repeat spacing D which contains a bilayer and its associated water. Previous studies obtained the mole ratio $\mathrm{n}_{\mathrm{W}}$ of water/lipid for fully hydrated D spacings (Nagle and Tristram-Nagle, 2000). For samples with different D spacings, $n_{W}$ was calculated, using the previously established result that the area per lipid remains the same within the investigated D spacing range (Nagle and TristramNagle, 2000). Dividing the sugar/lipid ratio $\mathrm{n}_{\mathrm{S}}$ by $\mathrm{n}_{\mathrm{W}}$ gave the sugar/water ratio that is converted to aqueous sugar concentration $\mathrm{C}_{S}$, listed in Table 1. This concentration is an average concentration of sugar in the water that includes both the water in the interfacial headgroup region and in the ample water space between neighboring bilayers in the well hydrated bilayer stacks.

Table 1. List of lipid/sugar samples and their exposures at different repeat spacings $\mathrm{D}$, corresponding to sugar concentrations $\mathrm{C}_{\mathrm{S}}$. Fully hydrated $\mathrm{D}$ values are indicated by ${ }^{*}$, and w estimates the relative goodness of the tilt-independent fit to the different exposures.

\begin{tabular}{|c|c|c|c|c|c|}
\hline Lipid & Sugar & $\mathrm{n}_{\mathrm{S}}$ & $\mathrm{D}(\AA)$ & $\mathrm{C}_{\mathrm{S}}(\mathrm{mM})$ & $w$ \\
\hline \multirow[t]{8}{*}{ SOPC } & none & 0 & $65.8^{*}$ & 0 & 0.33 \\
\hline & \multirow[t]{3}{*}{ Sucrose } & \multirow[t]{3}{*}{0.21} & 65.0 & 428 & 0.26 \\
\hline & & & 70.9 & 343 & 0.34 \\
\hline & & & 70.5 & 348 & 0.31 \\
\hline & \multirow[t]{2}{*}{ Glucose } & \multirow[t]{2}{*}{0.22} & 68.6 & 395 & 0.49 \\
\hline & & & 74.2 & 328 & 0.28 \\
\hline & \multirow[t]{2}{*}{ Fructose } & \multirow[t]{2}{*}{0.22} & 65.8 & 440 & 0.27 \\
\hline & & & 73.8 & 332 & 0.15 \\
\hline \multirow[t]{6}{*}{ DOPC } & none & 0 & $63.5^{*}$ & 0 & 0.33 \\
\hline & \multirow[t]{2}{*}{ Sucrose } & \multirow[t]{2}{*}{0.21} & 65.6 & 368 & 0.26 \\
\hline & & & 69.3 & 323 & 0.29 \\
\hline & \multirow[t]{3}{*}{ Fructose } & \multirow[t]{3}{*}{0.21} & 65.9 & 364 & 0.13 \\
\hline & & & 67.0 & 349 & 0.13 \\
\hline & & & 62.0 & 425 & 0.29 \\
\hline
\end{tabular}




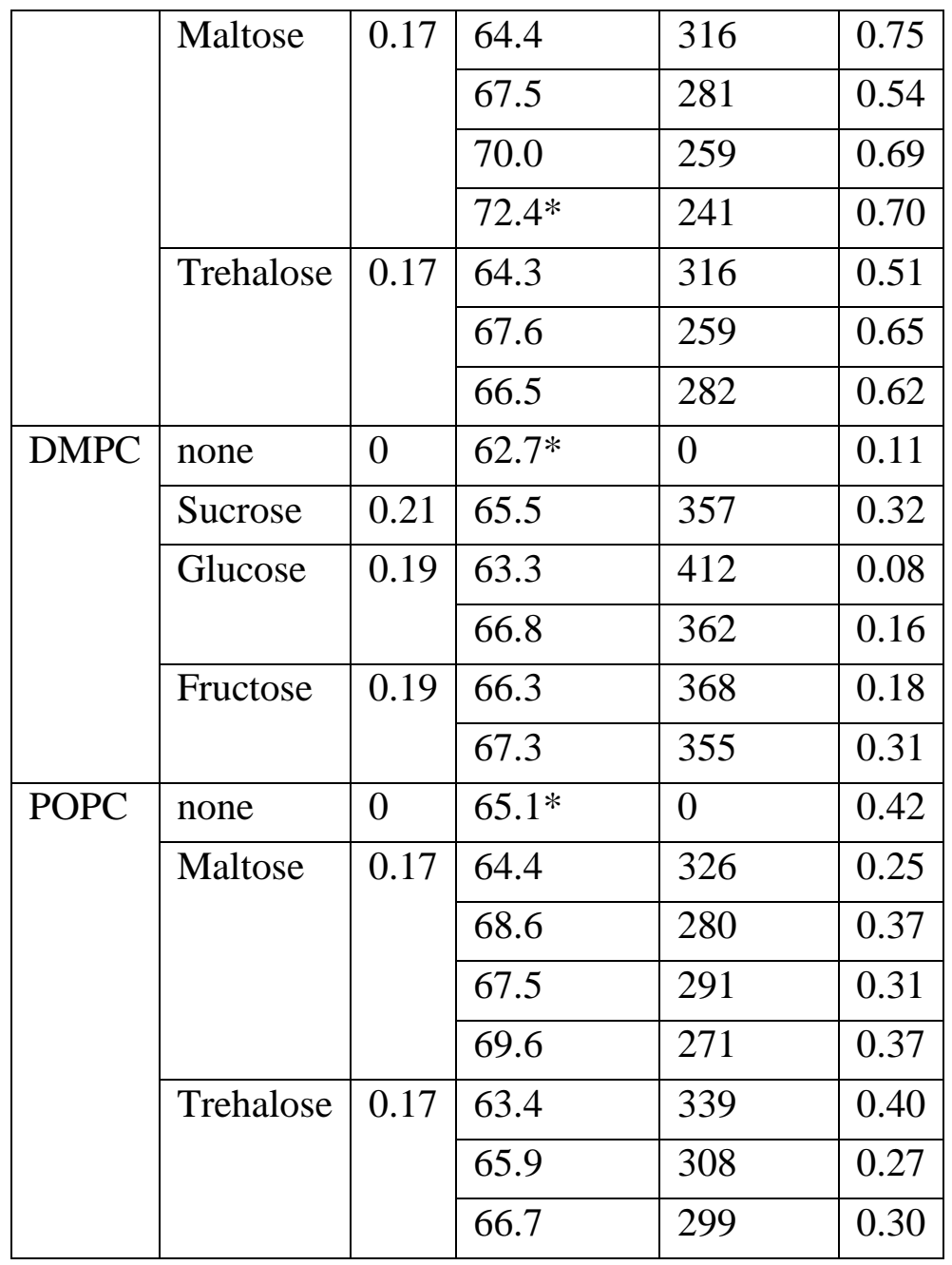

X-ray scattering data were taken at G1 station at the Cornell High Energy Synchrotron Source (CHESS), following published protocols (Liu and Nagle, 2004). A sample was placed in a hydration chamber maintained at $30^{\circ} \mathrm{C}$, and it was hydrated through the vapor phase. All bilayers were in the fluid phase (relative humidity > 99\%) for all reported results. The x-ray wavelength was either $1.177 \AA$ or $1.108 \AA$. During an $\mathrm{X}$-ray exposure, the incident angle was continuously varied by rotating the sample between $-1.6^{\circ}$ and $7^{\circ}$. The lamellar repeat $\mathrm{D}$ spacing was increased by increasing the current through a Peltier cooler in contact with the bottom of the silicon wafer holding the sample; this cooling of the sample compared to the vapor increased the effective relative humidity at the sample. Diffuse scattering data were fit using a new analysis method that obtains both the bending modulus $\mathrm{K}_{\mathrm{C}}$ and the tilt modulus $\mathrm{K}_{\theta}$ as the 
parameters that provide the best fit to the measured intensity (Jablin et al., 2014, Jablin, 2015). The data were also fit with $\mathrm{K}_{\theta}$ fixed to a very large value, thereby effectively removing tilt from the analysis; these tilt-independent results for $\mathrm{K}_{\mathrm{C}}$ agreed well with the earlier analysis method that did not incorporate tilt in the elasticity model (Liu and Nagle, 2004). Some samples were better fit than others; the inverse of the root mean residual sum of squares was used to assign a relative weight with values w shown in Table 1. 


\section{Results}

Table 1 lists samples analyzed for this study. Most combinations of lipid and sugar were measured with several different values of the repeat spacing D in order to obtain several sugar concentrations $\mathrm{C}_{\mathrm{S}}$ for the same sample. Usually, the sample was allowed to gradually become more hydrated, although some decreases in D were deliberately induced by manipulating the Peltier current. The time sequence for the exposures of each lipid/sugar sample followed the order shown in Table 1. For the controls with no sugar, the values for the D spacing shown in Table 1 are the fully hydrated values, $\mathrm{D}^{*}$, that have been well established in these and previous studies (Nagle and Tristram-Nagle, 2000). For some of the sugar samples, it was verified that the largest reported D spacing was near its fully hydrated value $\mathrm{D}^{*}$, although time did not permit accurate determinations of $\mathrm{D}^{*}$ for all samples. Nevertheless, we estimate for the concentrations of sugar shown in Table 1 that the fully hydrated $\mathrm{D}^{*}$ for the DMPC samples was about $69 \AA$, for DOPC samples D* was about $73 \AA$, for POPC samples D* was about $72 \AA$, and for SOPC samples D* was about $75 \AA$. The important result is that for all cases the samples with sugar swelled to larger D values than the control with no sugar.

Figure 1 shows results for the bending modulus $\mathrm{K}_{\mathrm{C}}$ that were determined by fitting the data using the tilt-independent theory. To efficiently show the overall effect of all the sugars on all the lipids, the $\mathrm{K}_{\mathrm{C}}$ for each lipid with sugar was divided by $\mathrm{K}_{\mathrm{C}}$ for the pure lipid control. As emphasized earlier (Nagle et al., 2015), the DOPC/glucose ratios were all close to 1 , with no systematic variations with sugar concentration $\mathrm{C}_{\mathrm{S}}$. Other results are more scattered. The smallest ratios are for DOPC/trehalose, but, contrarily, these trend to larger, not smaller, ratios with increasing $\mathrm{C}_{\mathrm{S}}$. The concentration dependence of trehalose appears different from the other sugars, as highlighted in Figure $\mathrm{S} 1$ in supplementary content where it is noted that this result doesn't seem to relate to trehalose having special cryoprotectant properties. There is also no significant trend to smaller ratios with increasing $\mathrm{C}_{\mathrm{S}}$ for the other lipid/sugar samples. The aggregate of DMPC ratios are greater than 1; this came about because the value of $\mathrm{K}_{\mathrm{C}}$ for the pure DMPC control was smaller compared to previous results from this lab (Kucerka et al., 2005). Generally, our results for $\mathrm{K}_{\mathrm{C}}$ for a single sample are subject to a standard 
deviation upwards to $10 \%$, so up to $20 \%$ deviation in the ratios of two samples was expected. Within this expected uncertainty, there is no significant indication in Fig. 1 of any sugar having a different effect than the others. Averaging over all ratios for all combinations was done in two ways. The average that used the relative weights in the last column of Table 1 gives a mean ratio of $0.978 \pm 0.015$ where 0.015 is the estimated error of the mean, not the standard deviation. Averaging over all results with equal weights gives a mean ratio of $1.005 \pm 0.015$. Both averages are consistent with insignificant effect of sugar on $\mathrm{K}_{\mathrm{C}}$.

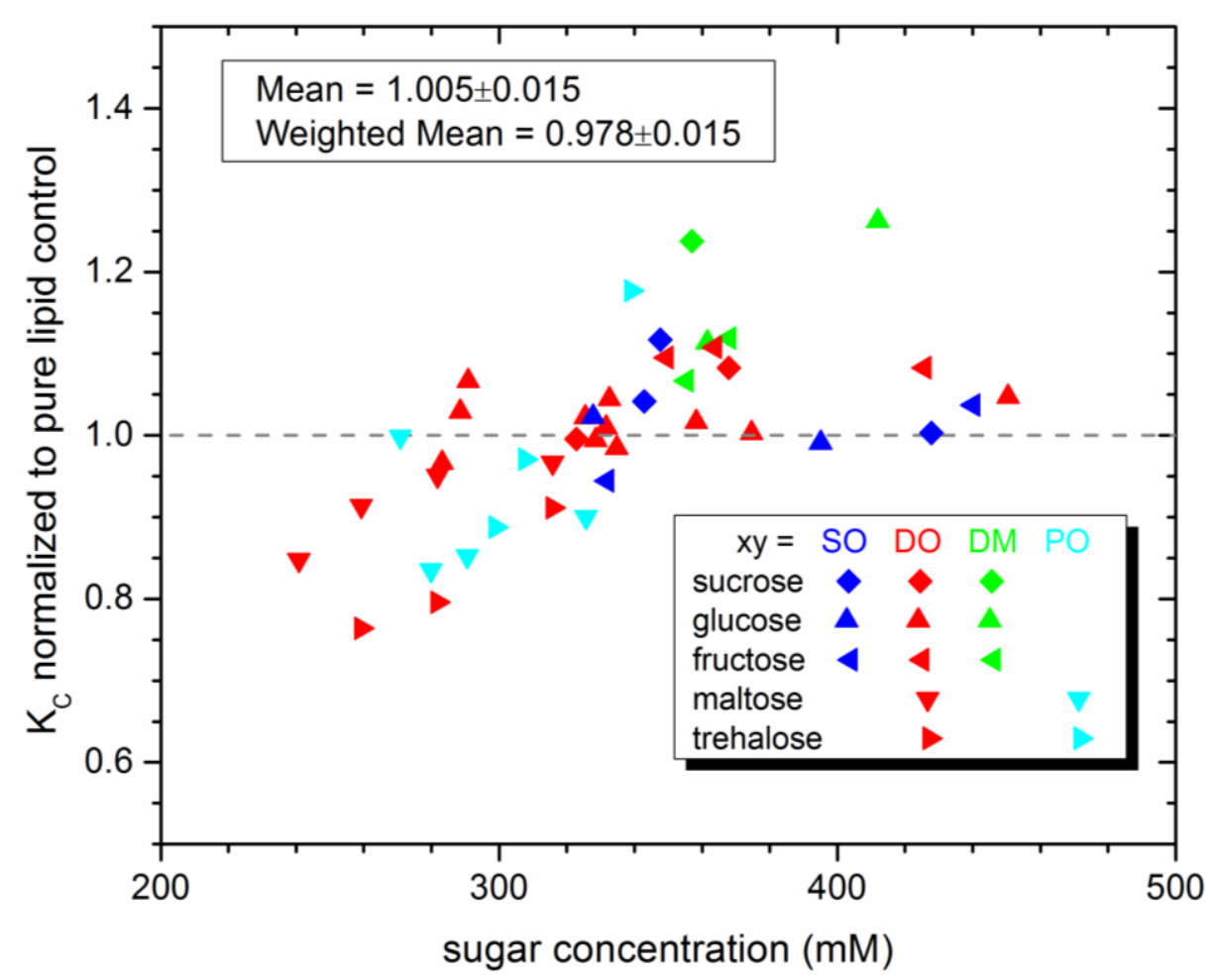

Figure 1: Normalized $\mathrm{K}_{\mathrm{C}}$ values for the combination of the sugars listed in the rows of the legend and for the xyPC lipids indicated in the columns of the legend. Normalization was to each pure lipid control. Values were obtained from tiltindependent fits. The DOPC/glucose results were previously published (Nagle et al., 2015).

Figure 2 shows similar results to Fig. 1 but for the tilt-dependent fits. The standard deviation of normalized $\mathrm{K}_{\mathrm{C}}(0.153)$ is somewhat greater than for the tilt- 
independent fits (0.105), but the unweighted mean and the estimated error of the mean are consistent with the results of the tilt-independent fits.

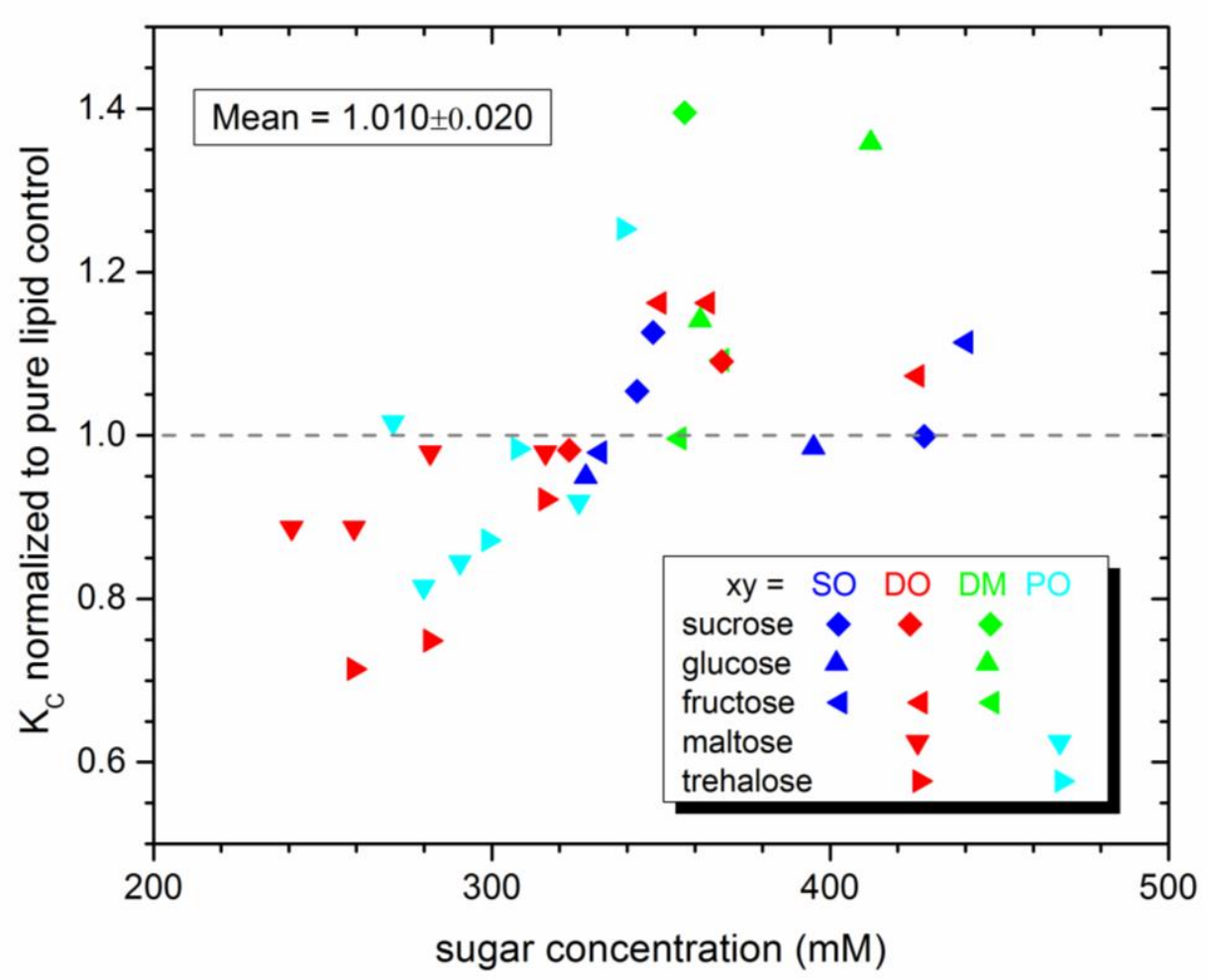

Figure 2: Values obtained from the tilt-dependent fits for the normalized $\mathrm{K}_{\mathrm{C}}$ values for the combination of the sugars listed in the rows of the legend and for the xyPC lipids indicated in the columns of the legend. Normalization was to each pure lipid control.

The tilt-dependent analysis also determines the tilt modulus $\mathrm{K}_{\theta}$. Figure 3 shows the ratios of $K_{\theta}$ with and without sugar. The relative standard deviation for $K_{\theta}$ is greater than for $\mathrm{K}_{\mathrm{C}}$ as is expected because the diffuse scattering intensity is much more sensitive to the value of $\mathrm{K}_{\mathrm{C}}$ than to $\mathrm{K}_{\theta}$. (However, tilt and bending play partially compensatory roles, so the larger uncertainty in $\mathrm{K}_{\theta}$ lends an additional uncertainty to the values of $\mathrm{K}_{\mathrm{C}}$ in the tilt-dependent fits; this partially accounts for the somewhat larger standard deviation in Fig. 2 compared to Fig. 1.) As with $K_{C}$, it appears that there is no systematic effect of sugar concentration on $\mathrm{K}_{\theta}$. 


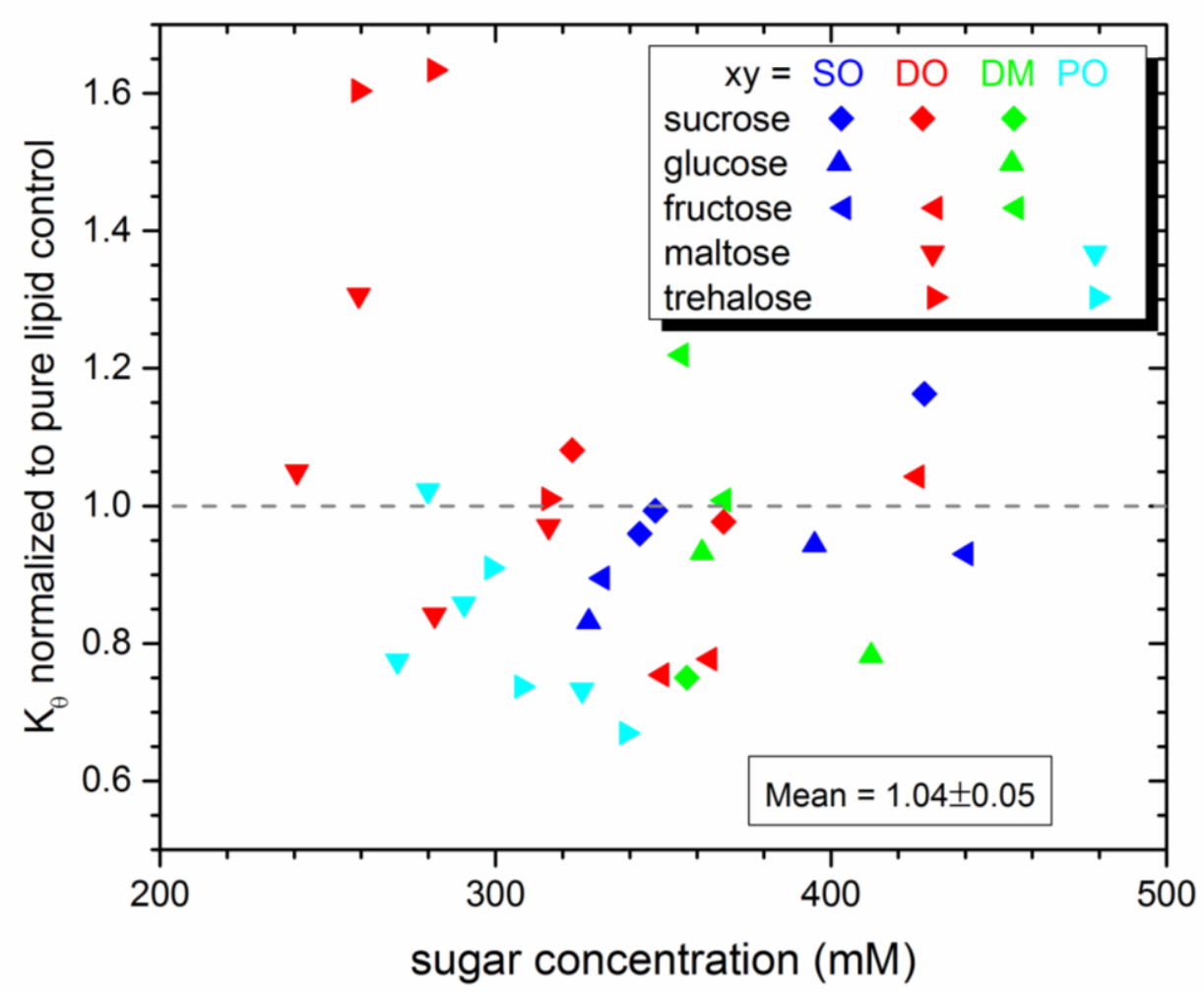

Figure 3: Normalized values of the tilt modulus $\mathrm{K}_{\theta}$ for the combination of the sugars listed in the rows of the legend and for the xyPC lipids in the columns of the legend. Normalization was to each pure lipid control.

\section{Discussion and Conclusions}

Our results show that there is no effect of common sugars on the bending modulus $\mathrm{K}_{\mathrm{C}}$ or the tilt modulus $\mathrm{K}_{\theta}$ of common lipids when determined using our $\mathrm{x}$-ray scattering method. This is the first study of the effect of sugar on the tilt modulus $\mathrm{K}_{\theta}$.

Since our bending modulus result disagrees with some reported results using other methods (Nagle, 2013), let us discuss possible causes. The most obvious a priori cause for our not observing an effect might have been that sugar was not well incorporated into our samples, but was sequestered in regions where it did not significantly interact with the stacks of bilayers. Although we had limited control over this, fortunately, it was clearly not the case because sugar caused the repeat D spacing to increase dramatically. If the sugar had remained outside the stack of bilayers, then it would either have not affected the bilayers in which case D would have remained the same, or sugar would 
have competed with the bilayers for water, causing the D spacing to decrease. Another possible reason might have been that sugar could have been very strongly bound to the interfacial region so the average sugar concentrations we obtained would effectively have corresponded to negligible bulk concentrations in giant unilamellar vesicles used for the classical experimental methods. As discussed previously (Nagle et al., 2015), based on the partition coefficient of sugar between bulk water and bilayers (Andersen et al., 2011), the bulk water concentration is unlikely to be smaller by more than a factor of two. The average concentrations shown in Table 1 were chosen to be larger than most concentrations (10-300 mM) employed in giant unilamellar studies in order to ensure that, even if our effective concentrations were smaller than the nominal values by a factor of two, they would still be as large as those typically used.

The effect of sugar on the bending modulus as observed by the classical methods might be hypothesized as being due to $\mathrm{K}_{\mathrm{C}}$ actually having different values on different length scales. Our x-ray analysis is more sensitive to shorter length scales of order 10$100 \AA$. In contrast, both classical methods assess longer length scales than the x-ray method; the length scale of the shape analysis method is of order of $10^{4}-10^{5} \AA$, and the mechanical manipulation method averages from $10^{5} \AA$ down to $10 \AA$. We will now argue that our result that sugar does not affect the $\mathrm{X}$-ray value of $\mathrm{K}_{\mathrm{C}}$ is inconsistent with the hypothesis that $\mathrm{K}_{\mathrm{C}}$ depends on the length scale. First, consider the ratios $\mathrm{n}_{\mathrm{S}}$ of sugar molecules to lipid molecules given in Table 1; these correspond to about one sugar for five lipid molecules. This number of lipids has an interfacial area of approximately 5(65 $\AA^{2}$ ) = 325 $\AA^{2}$, corresponding to a square of side length $\sim 18 \AA$. This surface coverage of sugar is well characterized as homogeneous for the two classical methods, whereas it is within the range of x-ray sensitive length scales. Sugar could therefore have a different effect on the $\mathrm{x}$-ray determined $\mathrm{K}_{\mathrm{C}}$ values which we now discuss. Each sugar molecule might induce a local perturbation in the bilayer height profile that would surely be limited to $1 \mathrm{~nm}$. The SA method looks at long wavelength thermal undulations and those have much larger amplitudes than $1 \mathrm{~nm}$. Therefore, the local $1 \mathrm{~nm}$ perturbations just roughen those undulations on a length scale much shorter than the resolution limit of a typical optical microscope. In contrast, the shorter wavelength undulations assessed by x-rays have much smaller amplitudes. The putative local perturbations would substantially 
increase the relative amplitudes of these short wavelength modes and that increase would be captured by the $\mathrm{x}$-ray analysis as a reduction in $\mathrm{K}_{\mathrm{C}}$ even greater than a possible reduction due to sugar softening the bilayer. Our x-ray result of no reduction of $K_{C}$ due to sugar is inconsistent with either type of reduction occurring. It therefore seems that the length scale hypothesis does not reconcile our X-ray results with previous reports that sugar reduces $\mathrm{K}_{\mathrm{C}}$.

The time scale is another difference between methods. Of course, $\mathrm{K}_{\mathrm{C}}$ is an equilibrium property that therefore can not depend upon the time scale for systems which are in equilibrium, unless there is an artifact in the measurement method. The measured $\mathrm{X}$-ray intensities are an ensemble average over many states. Each state is an average over the photon time scale of order femtoseconds, far too short for any significant temporal averaging of the sample to occur. Temporal averaging should also not be a concern for micromanipulation measurements which only assess the average area. However, it has been recognized to be a concern for the shape analysis method with its millisecond averaging (Faucon et al., 1989, Drabik et al., 2016). Temporal averaging smooths a vesicle's contour and such smoothing could be interpreted artifactually as larger values of $\mathrm{K}_{\mathrm{C}}$. (The obvious extreme example is that if one waits long enough, the vesicle shape averages to a sphere which would correspond to an infinite value of $\mathrm{K}_{\mathrm{C}}$.) Sugar increases the viscosity, thereby slowing down the fluctuations. For a given experimental apparatus with a fixed time scale, there would then be less temporal averaging with sugar, and if appropriate corrections were not made, the apparent value of $\mathrm{K}_{\mathrm{C}}$ would appear smaller than its value without sugar, even if there were no real difference.

In conclusion, we find no effect of sugars on the bending modulus $K_{C}$ or on the tilt modulus $\mathrm{K}_{\theta}$ of neutral PC lipid bilayers using our $\mathrm{x}$-ray method. In view of this, we urge further study using other methods to address whether there is an effect of sugar on the bending modulus.

Acknowledgements: We thank beamline scientist Arthur Woll for facilitating our collecting x-ray scattering data at the G1 station of the Cornell High Energy Synchrotron Source (CHESS), which is supported by the National Science Foundation and the National Institutes of Health/National Institute of General Medical Sciences under 
National Science Foundation Award DMR-0225180. This research was supported in part by Grant No. GM 44976 from NIGMS/NIH; the content is solely the responsibility of the authors and does not necessarily represent the official views of the National Institutes of Health.

\section{Bibliography}

ANDERSEN, H. D., WANG, C. H., ARLETH, L., PETERS, G. H. \& WESTH, P. 2011. Reconciliation of opposing views on membrane-sugar interactions. Proceedings of the National Academy of Sciences of the United States of America, 108, 18741878.

BOUVRAIS, H. 2012. Bending Rigidities of Lipid Bilayers: Their Determination and Main Inputs in Biophysical Studies. In: IGLIČ, A. \& GENOVA, J. (eds.) Advances in Planar Lipid Bilayers and Liposomes.: Academic Press.

DRABIK, D., PRZYBYLO, M., CHODACZEK, G., IGLIC, A. \& LANGNER, M. 2016. The modified fluorescence based vesicle fluctuation spectroscopy technique for determination of lipid bilayer bending properties. Biochim. Biophys. Acta Biomembranes, to be published.

EVANS, E. \& RAWICZ, W. 1990. Entropy-driven tension and bending elasticity in condensed-fluid membranes Physical Review Letters, 64, 2094-2097.

FAUCON, J. F., MITOV, M. D., MELEARD, P., BIVAS, I. \& BOTHOREL, P. 1989. Bending elasticity and thermal fluctuations of lipid membranes. Theoretical and experimental requirements. J. de Physique, 50, 2389-2414.

GRACIA, R. S., BEZLYEPKINA, N., KNORR, R. L., LIPOWSKY, R. \& DIMOVA, R. 2010. Effect of cholesterol on the rigidity of saturated and unsaturated membranes: fluctuation and electrodeformation analysis of giant vesicles. Soft Matter, 6, 1472-1482.

HENRIKSEN, J. R. \& IPSEN, J. H. 2002. Thermal undulations of quasi-spherical vesicles stabilized by gravity. European Physical Journal E, 9, 365-374.

HENRIKSEN, J. R. \& IPSEN, J. H. 2004. Measurement of membrane elasticity by micro-pipette aspiration. European Physical Journal E, 14, 149-167.

JABLIN, M. S. 2015. Tilt-Dependent Analysis of Diffuse X-ray Scattering from Oriented Stacks of fluid Phase Lipid Bilayers. http://lipid.phys.cmu.edu: Thesis.

JABLIN, M. S., AKABORI, K. \& NAGLE, J. F. 2014. Experimental Support for TiltDependent Theory of Biomembrane Mechanics. Physical Review Letters, 113, $248102(1-5)$.

KUCERKA, N., LIU, Y. F., CHU, N. J., PETRACHE, H. I., TRISTRAM-NAGLE, S. T. \& NAGLE, J. F. 2005. Structure of fully hydrated fluid phase DMPC and DLPC lipid bilayers using X-ray scattering from oriented multilamellar arrays and from unilamellar vesicles. Biophysical Journal, 88, 2626-2637.

LI, D. P., HU, S. X. \& LI, M. 2006. Full q-space analysis of X-ray scattering of multilamellar membranes at liquid-solid interfaces. Physical Review E, 73, 031916. 
LIU, Y. F. \& NAGLE, J. F. 2004. Diffuse scattering provides material parameters and electron density profiles of biomembranes. Physical Review E, 69, 040901.

LYATSKAYA, Y., LIU, Y. F., TRISTRAM-NAGLE, S., KATSARAS, J. \& NAGLE, J. F. 2001. Method for obtaining structure and interactions from oriented lipid bilayers. Physical Review E, 63, 011907.

MELEARD, P., GERBEAUD, C., BARDUSCO, P., JEANDAINE, N., MITOV, M. D. \& FERNANDEZ-PUENTE, L. 1998. Mechanical properties of model membranes studied from shape transformations of giant vesicles. Biochimie, 80, 401-413.

MELEARD, P., GERBEAUD, C., POTT, T., FERNANDEZPUENTE, L., BIVAS, I., MITOV, M. D., DUFOURCQ, J. \& BOTHOREL, P. 1997. Bending elasticities of model membranes: Influences of temperature and sterol content. Biophysical Journal, 72, 2616-2629.

NAGLE, J. F. 2013. Introductory Lecture: Basic quantities in model biomembranes. Faraday Discussions, 161, 11-29.

NAGLE, J. F., JABLIN, M. S., TRISTRAM-NAGLE, S. \& AKABORI, K. 2015. What are the true values of the bending modulus of simple lipid bilayers? Chemistry and Physics of Lipids, 185, 3-10.

NAGLE, J. F. \& TRISTRAM-NAGLE, S. 2000. Structure of lipid bilayers. Biochimica Et Biophysica Acta-Reviews on Biomembranes, 1469, 159-195.

PAN, J. J., TRISTRAM-NAGLE, S., KUCERKA, N. \& NAGLE, J. F. 2008.

Temperature dependence of structure, bending rigidity, and bilayer interactions of dioleoylphosphatidylcholine bilayers. Biophysical Journal, 94, 117-124.

PAN, J. J., TRISTRAM-NAGLE, S. \& NAGLE, J. F. 2009. Effect of cholesterol on structural and mechanical properties of membranes depends on lipid chain saturation. Physical Review E, 80, 021931.

PECREAUX, J., DOBEREINER, H. G., PROST, J., JOANNY, J. F. \& BASSEREAU, P. 2004. Refined contour analysis of giant unilamellar vesicles. European Physical Journal E, 13, 277-290.

RAWICZ, W., OLBRICH, K. C., MCINTOSH, T., NEEDHAM, D. \& EVANS, E. 2000. Effect of chain length and unsaturation on elasticity of lipid bilayers. Biophysical Journal, 79, 328-339.

SALDITT, T., VOGEL, M. \& FENZL, W. 2003. Thermal fluctuations and positional correlations in oriented lipid membranes. Physical Review Letters, 90, 178101.

SHCHELOKOVSKYY, P., TRISTRAM-NAGLE, S. \& DIMOVA, R. 2011. Effect of the HIV-1 fusion peptide on the mechanical properties and leaflet coupling of lipid bilayers. New Journal of Physics, 13, 025004.

TRISTRAM-NAGLE, S. 2007. Preparation of Oriented, Fully Hydrated Lipid Samples for Structure Determination Using X-Ray Scattering, Totowa, NJ, Humana Press.

VITKOVA, V., GENOVA, J., MITOV, M. D. \& BIVAS, I. 2006. Sugars in the aqueous phase change the mechanical properties of lipid mono- and bilayers. Molecular Crystals and Liquid Crystals, 449, 95-106.

VITKOVA, V. \& PETROV, A. G. 2013. Lipid bilayers and membranes: Material properties. In: IGLIČ, A. \& GENOVA, J. (eds.) Advances in Planar Lipid Bilayers and Liposomes.: Academic Press. 